\title{
The new era in office-based facial rejuvenation: Promising technology of silicone threads
}

\author{
Naci Celik ${ }^{* 1,2}$ iD \\ ${ }^{1}$ Istanbul Okan University, Faculty of Applied Sciences, Department of Gastronomy, 34959, Istanbul, Turkey \\ ${ }^{2}$ Plastic and Reconstructive Surgeon, Private Practice in Dr. Naci Celik's Clinic, 34367, Sisli, Istanbul, Turkey
}

\begin{abstract}
Aging is unpreventable, although its symptoms vary a lot among individuals because of the genetic determinants and one's life habits. Sun exposure, bad habits like excessive alcohol consumption, and smoking accelerate the aging process and urge people to seek for a solution to reverse the changes, especially for the most prominent part of our body, the face. Unfortunately, there is no one simple solution for that, and it includes a bunch of surgical and non-surgical interventions. Relatively simple methods have fewer risks, but the reversal effect is also minor. This includes neurotoxin and filler injections as well as energy-based devices. More competent surgical options, alas, come with a long and difficult recovery period and diverse, sometimes inevitable, complications. Most of the time, people are scared of the surgery and accept less invasive methods. Among these, thread lift is perceived as the missing link between the surgery and non-invasive methods. Unfortunately, up to recent years, the results of threads have not been promising, and they also have many complications. A new type of thread originated in France, made of silicone and polyester, gives promising results. This paper reviews the history and specifications of the threads and tries to explain the logic of their use in facial rejuvenation.
\end{abstract}

Keywords: Facelift; infinite-thread ${ }^{\mathbb{}}$; silicone threads; spring thread ${ }^{\mathbb{}}$; threadlift

\section{Introduction}

Aging is a degenerative process and a complex biological phenomenon caused by intrinsic and extrinsic factors (Charlesde-Sá et al., 2018). Intrinsic aging is largely genetically determined and affects the skin through a slow and partly reversible degeneration of connective tissue (Uitto et al., 1986). On the other hand, extrinsic aging, primarily ultraviolet radiation, results in premature aging even in young individuals (Scharffetter-Kochanek et al., 2000). The neck and face are sunexposed areas like hands. These areas are under an overlapping influence of intrinsic and extrinsic factors, which produces a more complex and faster aging process (Fisher et al., 2002). The symptoms of aging can be concealed in some areas, thanks to advanced surgical techniques (Rodriquez-Bruno and Papel,
2011), amazing hyaluronic and non-hyaluronic acid fillers (Tan and Kontis, 2015), neurotoxin treatments (Raspaldo, 2011), energy-based devices (DiBernardo et al., 2016), and evolving thread technologies (Rezaee Khiabanloo et al., 2019).

Aging also results in a deficiency of facial fat compartments (Cenkeri et al., 2020), which can easily be reversed with fat injections (Obagi and Willis, 2018) or facial fillers (Philipp-Dormston et al., 2020). On the other hand, neither fillers nor the energy-based devices cannot be used successfully to lift the descended parts of the face; however, they are great adjuncts following facelift to obtain a better aesthetic outcome (Hammoudeh and Stevens, 2019).

Surgery is usually the preferred choice of treatment for optimal results in face and neck rejuvenation. There are several surgical face and neck lift methods (Beaty, 2014). Although

${ }^{*}$ Corresponding author.

E-mail address: drnacicelik@gmail.com (N. Celik).

https://doi.org/10.51753/flsrt.866329 Author contributions

Received 22 January 2021; Accepted 5 March 2021

Available online 03 April 2021

(C) 2021 Dergipark. All rights reserved. 
surgery's success is not deniable in this area, there are particularly challenging cases either due to anatomical variations or psychological expectations based on the individual's perception of the problem and motivation for change. A thorough patient evaluation and counseling are mandatory before considering any surgical approach in the neck and face, and modifications of the surgical approach are needed, if necessary (Smith and Papel, 2018). On the other hand, surgery alone may fail to meet some neck problems, such as superficial aging changes, as it addresses excision only. Chromophorebased pathologies, vascular changes, epidermal and dermal nonchromophore-based lesions are among these problems (Mulholland, 2014). It is also important to individualize the relationship between the lower face, jawline, and neck (Celik, 2020a). As people age, some of the lower facial tissues descend beyond the jawline, which makes the correction of facial tissues crucial to accomplish a substantial improvement in the aesthetics of the neck (Rohrich et al., 2006). In brief, good results with face and neck rejuvenation can be achieved if it is combined with lower face and jawline procedures, filler injections, and the inclusion of energy-based therapies (Celik, 2020b).

Nonetheless, there are three important reasons for writing this paper;

1. Surgery offers its advantages together with its complications, like a longer recovery period and inevitable scars.

2. Not every doctor can perform this kind of surgery. This includes inexperienced plastic surgeons.

3. Not every patient is fond of surgery, and they are looking for the so-called "minimally invasive procedures".

Both patients and the doctors need a bridge between the surgery and non-surgical rejuvenation (Celik, 2020c). For more than two decades, thread lift methods tried to fill this gap, however in vain, most of the time.

\section{Historical background and classification}

\subsection{Non-absorbable polypropylene threads}

Thread lifting has come to its current place after a long journey (Savoia et al., 2014), which began in the nineties with non-absorbable polypropylene threads (Sulamanidze et al., 2002). The doctors' early excitement created popular officebased procedures called a lunch-time facelift by media (Atiyeh et al., 2010). However, they fell out of favor because of high complication rates and fast temporary results (Lycka et al., 2004). Most of the complications encountered were due to nonabsorbable suture material (Silva-Siwady et al., 2005). These types of complications necessitated surgical interventions to solve a problem of non-surgical intervention. Complications and lack of a good long-term effect of the threads were the most common reasons for the technique's abandonment (Rachel et al., 2010).

\subsection{Absorbable threads}

With the introduction of mixed absorbable and nonabsorbable threads and pure absorbable threads, thread lifting has again gained attention. Several manufacturing companies have produced diverse types of threads. Silhouette $\mathrm{Soft}^{\circledR}$ suture (Silhouette Soft ${ }^{\circledR}$, Sinclair Pharma GmbH, Irvine USA) was made of poly-L-lactic acid (PLLA) and consisted of multiple cones that were made of polylactide/glycolide copolymer
(PLGA). This thread was an absorbable one, and it was different from the other threads of the same company. Silhouette Lift ${ }^{\circledR}$ was composed of non-absorbable polypropylene suture and absorbable PLGA cones. Silhouette Instalift ${ }^{\circledR}$ contained only PLGA both in sutures and cones. PLLA and PLGA are biodegradable polymers, and PLLA is a well-known biomedical device for over four decades and has been used as absorbable plates, screws, and suture materials. PLLA triggers a foreign body reaction when implanted into the tissue. This reaction generates a cellular inflammatory response, which leads to the formation of vascularized, connective tissue (neocollagenesis) (Bohnert et al., 2019). This neocollagenesis, when coupled with repositioning by the sutures' cones, makes these kinds of suspension sutures a valuable tool for facial rejuvenation (Goldberg, 2020).

Another kind of absorbable thread that is used for facial rejuvenation is polydioxanone (PDO). Polydioxanone sutures were in plastic surgery as intradermal sutures since the ' $80 \mathrm{~s}$ (Chusak and Dibbell, 1983). Years later, originated in Asia, PDO threads became available for facial rejuvenation around 2015 (Suh et al., 2015). PDO is a synthetic polymer and absorbed from the body by hydrolyzing in 6 months. The addition of barbs to PDO tries to increase the load-bearing ability when used as suspension sutures. However, the aim is to lift the ptotic facial tissues, polydioxanone could not achieve this goal, and it is often used as "solid fillers" to treat deep static wrinkles on the face (Kang et al., 2019). Polydioxanone fills the wrinkle first by its volume and later by the mild local inflammatory reaction, which results in lymphocytic infiltration and subsequent fibrosis. Although there are many variations, polydioxanone threads for facial rejuvenation can be classified roughly into three different types:

1. Mono PDO thread: non-barbed and thin $(0.07-0.15 \mathrm{~mm})$ monofilament thread.

2. Spring or twin thread: Braided 2 monofilament PDOs or twined single monofilament. It is more tensile than mono PDO.

3. COG PDO thread: This one has barbs and creates a lifting effect when pulled. The cogs were shown to induce a fibrotic reaction four weeks after the insertion (Jang, 2005). Depending on the direction of the barbs, they can be categorized as:

a) Unidirectional.

b) Bidirectional.

c) Multidirectional.

Although absorbable threads were relatively well-accepted by dermatologists and aesthetic practitioners, plastic surgeons were distant from these kinds of procedures since the beginning. Many cosmetic companies produce their own so-called threadlifting sutures, and these companies have spent a great deal of marketing budget for the training of physicians and nonphysicians. In combination with enthusiastic cosmetic professionals, these commercial encouragements are mainly responsible for the extensive spread of this alleged "minimally invasive" lifting procedure. On the other hand, even the most optimistic non-surgeon doctors emphasized that thread lifting is neither an alternative to surgery nor magic per se, but it could have good results for rejuvenation and skin tightening, especially when combined with other tools of rejuvenation (Ali, 2018). Although the facelift effect seems very subtle, the complications of absorbable sutures are regarded as minimal or moderate without permanent sequela (Sarigul Guduk and Karaca, 2018). In another study, despite the results seemed to be 
good, the authors concluded that, because of the high complication rates of the PDO threads, short-lived benefits, and similar downtime and costs, traditional facelifting was to be preferred (Bertossi et al., 2019).

Most of the studies published show only a limited effect and longevity. A recent review of thread-lift sutures (Gulbitti et al., 2018) concludes that the use of threads seems to be promising when they are used in combination with an open procedure.

\subsection{Silicone threads}

Being a skeptic plastic surgeon, the author of this publication refused to use threads until 2016, when nonabsorbable silicone threads were introduced in the Turkish market (Spring Thread ${ }^{\circledR}$, 1st SurgiConcept 96 Rue de Pont, Rompu 59200 Tourcoing, France). Silicone threads do not rely on fibrosis and remodeling and subsequent skin tightening for lifting effect as absorbable threads do. Instead, their mechanism is simpler and more rational for a plastic surgeon; the lift effect is created by the upright movement of tissues, thanks to the barbs. Although the mechanism and logic were similar to primitive non-absorbable sutures, the technology and the material used are different. Silicone thread is a biocompatible composite material. The outer part is medical grade silicone, which envelopes the polyester inner part. It is elastic and can be elongated by $20 \%$. This elasticity provides a spring effect that compensates for the creep of classic threads. The author used this thread alone and in combination with surgery (Celik, 2020d). Although it is beyond this paper's scope, the main advantages and disadvantages of Spring Thread $^{\circledR}$ should be addressed concisely.

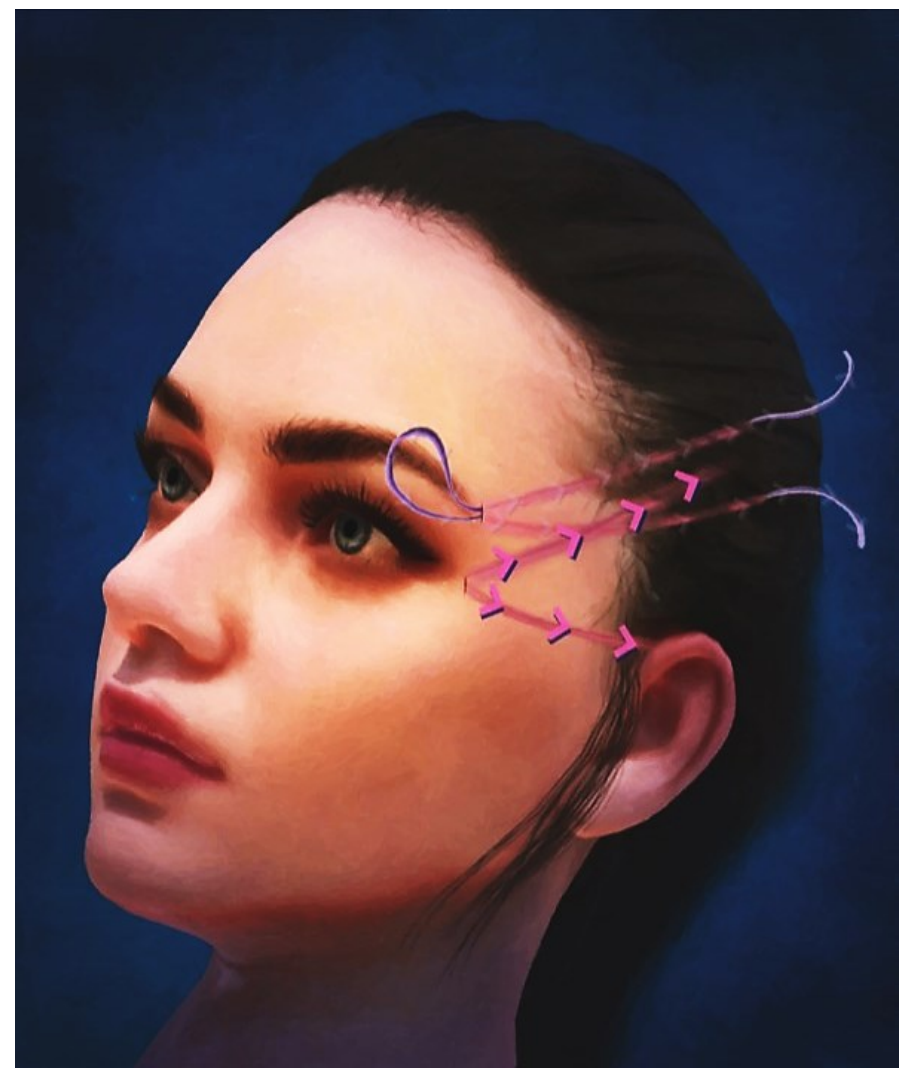

Fig. 1. Drawing of thread placement for browlift and canthopexy.

The usual thread reactions like extrusion or granuloma formation encountered in the polypropylene threads were not seen in silicone threads. The lifting effect was more powerful than the other barbed sutures that the author used before. On the other hand, slipping of the silicone coating from the polyester core during the thread's implantation was not unusual, necessitating another box of thread. According to the author, another problem was the elasticity of the silicone. However, in the beginning, it was presented as one of the advantages of this new type of thread; by the time, it was realized that the elasticity was one of the disadvantages of this thread. Briefly, elasticity causes shorter thread length and less amount of barbes inside the tissue. The thread shortens back to its unaltered length after some time, and the pull effect decreases. Later in 2019, After 2 years of experience with more than 200 patients, the author's perception about the threads was that they could not replace open-type face and neck lift surgeries but can be applied to many patients who are avoiding the complications of open surgery and seeking for some effect of a facelift for a few years at least. For this purpose, the author designed a new way of eyebrow lift and canthopexy instead of an endoscopic temporal lift (Fig. 1).

In 2019, the author started to use non-elastic silicone threads (Infinite-Thread ${ }^{\circledR}$, Thread \& Lift Laboratory, Brussels, Belgium). This one, just like the other silicone thread, is made in France, but the headquarter is in Brussels. After using InfiniteThread $^{\circledR}$ for a minimally invasive facelift, the author realized that this thread is a powerful game-changer. It has some unique properties which are different from the previous threads. It also has a polyester core coated with silicone. There are 4 cogs in every $1.5 \mathrm{~cm}$. Each series of cogs is offset at 45 degrees, and it creates an " 8 axis" hooking. The diameter is $1.4 \mathrm{~mm}$ from cog to

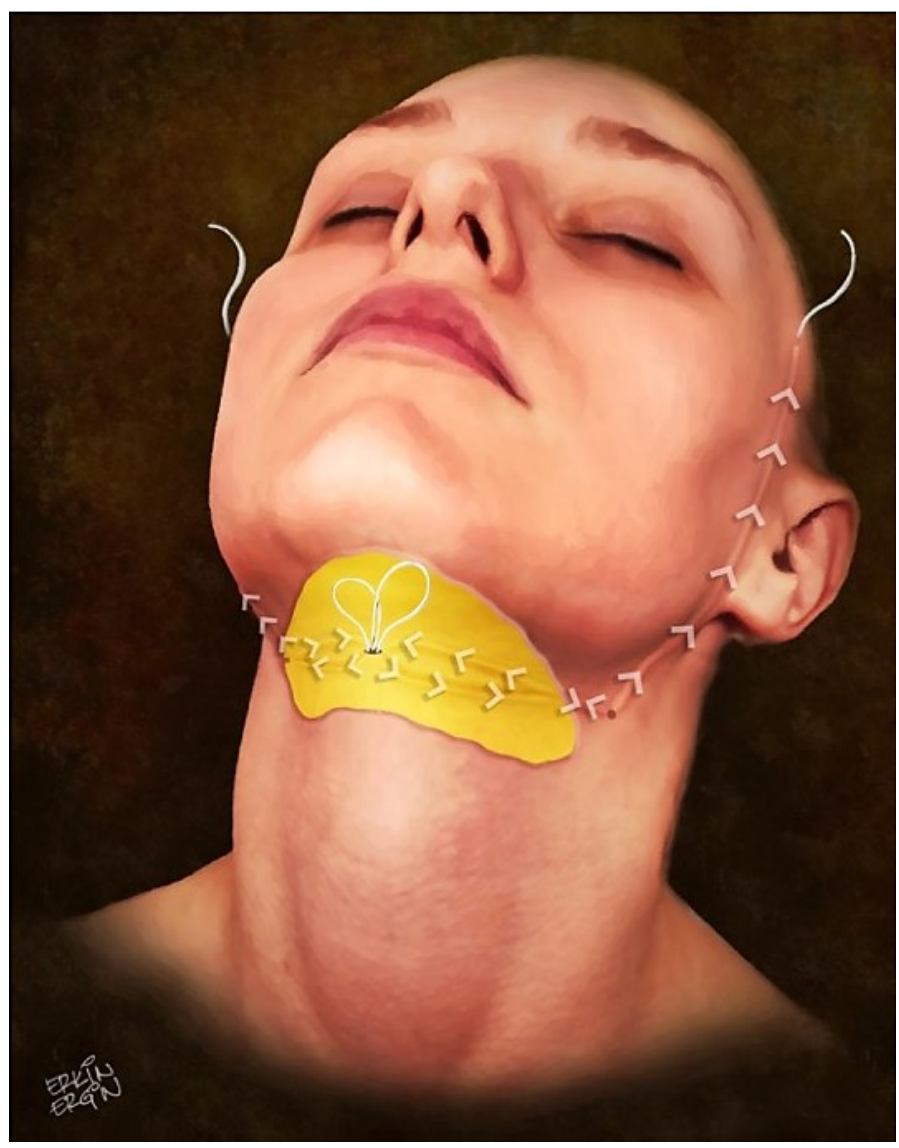

Fig. 2. Schematic representation of neck lift with silicone thread.

cog, but the cylinder of silicone is $0.5 \mathrm{~mm}$. Cogs have a unique design that prevents them from turning and slipping from the 
tissue thanks to their conical shape, which reinforces the cog at the base. Cogs have rounded tips that prevent the cheese-wire effect (a common problem of early polypropylene threads). Because of this new thread's powerful lift effect, a neck lift is also possible (Fig. 2). Infinite-Thread ${ }^{\circledR}$ is not elastic deliberately (does not elongate) but flexible.

There are not a lot of scientific articles about the results of silicone threads for facial rejuvenation. Although according to the author of this paper, non-elastic silicone thread seems promising, we need more studies to see their long-term effects and their capacity to lift the face.

\section{Discussion}

The minimal invasive facial rejuvenation concept is tempting for patients. It has gained high popularity among physicians and patients looking for an easy facial lifting method and skin rejuvenation. Although the plastic surgeons' curiosity has withered because of the fast-temporary effect and a bunch of complications of the threads, the patients have never lost interest, thanks to cosmetic doctors who offer the procedure (Celik and Gok, 2020). On the other hand, the literature review shows no evidence to support the threads' effectiveness unless studies are supported by industry.

We encounter many rumors of perfect lunch-time facelifts, websites promising perfect results, media interviews, and case reports. However, we do not come across detailed scientific papers about the results, long-term follow-ups, and other types of information that we used to see in scientific publications on other subjects. That is why plastic surgeons have lost their trust in threads, no matter what the material or the technique is. There are rare but promising publications about thread use in open surgeries published by surgeons (Matarasso, 2013; O'Connell, 2015). A combination of threads with other methods of facial rejuvenation also seems promising (Celik, 2020e). Nevertheless, the success of these encouraging results is not only because of the threads but because of the other techniques used with the threads, and they are far away from being a minimally invasive office procedure. On the other hand, recent developments in thread technology reveal a new era in facial rejuvenation. Under local anesthesia, office procedures may have a similar rejuvenating effect to those of open surgeries with minimal downtime and less risk of serious complications with these new silicone threads. This is especially very important for plastic surgeons who are strictly adhered to conventional methods. There is a publication from 2008 by D'Amico et al. which had seen the future (that future is our today now) and warned the plastic surgeons about the imminent danger of negligence. Their paper clearly shows the trend of the consumers (patients), plastic surgeons and non-plastic surgeon core providers (dermatologists, ENT specialists), and non-core providers (the rest of all cosmetic procedure providers) at that time. One vital message that can be learned from this study is that the patients would choose plastic surgeons to perform more invasive procedures $(90 \%)$, but the percentage decreases rapidly when it comes to less invasive (40\%) and the least invasive procedures $(15 \%)$. Another important note is that of consumers who had had a positive experience with a non-plastic surgeon for a noninvasive procedure, 47 percent said that the same provider would be their first choice for an invasive procedure (D'Amico et al., 2008). This means that should the plastic surgeons keep the distance to these minimally invasive methods, their number of surgical cases may drop over time.

\section{Conclusion}

Thread lifting is not a complication-free nor a minimal invasive lunch-time beautification procedure. Quite the contrary, it is not different from surgery in terms of complications, technical demands, and effects. Then why should we choose thread lift instead of surgery? The author of this paper thinks that we should not adhere to one of them. We should choose the right technique for the right patient. The decision should be made by a mutual agreement between the doctor and the patient. We should quit promoting surgery while vilifying thread lifts. One of our latest unpublished studies shows that $75 \%$ of patients who ask for thread lift and are refused by the doctor and offered surgery would find another doctor who would do the thread lift. More than half of the remaining 25\% forgoes any procedure. Plastic surgeons should overcome their prejudice and give another chance to evolving thread technology.

Conflict of interest: The author declares that they have no conflict of interests.

Informed consent: This manuscript did not involve human or animal participants; therefore informed consent was not collected.

\section{References}

Ali, Y. H. (2018). Two years' outcome of thread lifting with absorbable barbed PDO threads: Innovative score for objective and subjective assessment. Journal of Cosmetic and Laser Therapy, 20(1), 41-49.

Atiyeh, B. S., Dibo, S. A., Costagliola, M., \& Hayek, S. N. (2010). Barbed sutures "lunch time" lifting: evidence-based efficacy. Journal of cosmetic dermatology, 9(2), 132-141.

Beaty, M. M. (2014). A progressive approach to neck rejuvenation. Facial Plastic Surgery Clinics, 22(2), 177-190.

Bertossi, D., Botti, G., Gualdi, A., Fundarò, P., Nocini, R., Pirayesh, A., \& van der Lei, B. (2019). Effectiveness, longevity, and complications of facelift by barbed suture insertion. Aesthetic surgery journal, 39(3), 241-247.

Bohnert, K., Dorizas, A., Lorenc, P., \& Sadick, N. S. (2019). Randomized, controlled, multicentered, double-blind investigation of injectable Poly-L-Lactic Acid for improving skin quality. Dermatologic Surgery, 45(5), 718-724.

Celik, N. (2020a). Turkey neck no, but Turkish neck yes. Abstract book main session of AMWC 2020 (Aesthetic \& Anti-Aging Medicine World Congress), Monte-Carlo, Monaco. 5.
Celik, N. (2020b). How to create a bichatectomy look with threads and fillers. Abstract book main session of AMWC 2020 (Aesthetic \& Anti-Aging Medicine World Congress), Monte-Carlo, Monaco. 5.

Celik, N. (2020c). Surgical face lift or thread lift? Should we choose the combination? Abstract book main session of AMWC 2020 (Aesthetic \& Anti-Aging Medicine World Congress), Monte-Carlo, Monaco. 4.

Celik, N. (2020d). Silikon askılar ile cerrahi olmayan bişektomi görünümü. Dermatolojide \& Kozmetolojide Gelişmeler Kongresi Kongre Ekitab1. Istanbul, Turkey. 16.

Celik, N. (2020e). New combinations of threads with surgical methods for facial rejuvenation new combinations of threads with surgical methods for facial rejuvenation. Turkish Journal of Plastic Surgery, 28(4), 219-223.

Celik, N., \& Gok, I. (2020). Jawline Beautification with Threads: An easy office procedure. Abstract Book of 4th International Cosmetic Congress (Main Theme Ethnocosmetic) Istanbul, Turkey.

Cenkeri, H. C., Guduk, S. S., \& Cicek, E. D. (2020). Aging Changes of the Superficial Fat Compartments of the Midface Over Time: A Magnetic Resonance Imaging Study. Dermatologic Surgery, 46(12), 
1600-1605.

Charles-de-Sá, L., Gontijo-de-Amorim, N. F., Takiya, C. M., Borojevic, R., Benati, D., Bernardi, P., ... \& Rigotti, G. (2018). Effect of use of platelet-rich plasma (PRP) in skin with intrinsic aging process. Aesthetic Surgery Journal, 38(3), 321-328.

Chusak, R. B., \& Dibbell, D. G. (1983). Clinical experience with polydioxanone monofilament absorbable sutures in plastic surgery. Plastic and Reconstructive Surgery, 72(2), 217-221.

D’Amico, R. A., Saltz, R., Rohrich, R. J., Kinney, B., Haeck, P., Gold, A. H., ... \& Eaves III, F. (2008). Risks and opportunities for plastic surgeons in a widening cosmetic medicine market: future demand, consumer preferences, and trends in practitioners' services. Plastic and Reconstructive Surgery, 121(5), 1787-1792.

DiBernardo, B. E., DiBernardo, G., \& Pozner, J. N. (2016). Subsurface laser and radiofrequency for face and body rejuvenation. Clinics in Plastic Surgery, 43(3), 527-533.

Fisher, G. J., Kang, S., Varani, J., Bata-Csorgo, Z., Wan, Y., Datta, S., \& Voorhees, J. J. (2002). Mechanisms of photoaging and chronological skin aging. Archives of Dermatology, 138(11), 1462-1470.

Goldberg, D. J. (2020). Stimulation of collagenesis by poly-L-lactic acid (PLLA) and-glycolide polymer (PLGA)-containing absorbable suspension suture and parallel sustained clinical benefit. Journal of Cosmetic Dermatology, 19(5), 1172-1178.

Gulbitti, H. A., Colebunders, B., Pirayesh, A., Bertossi, D., \& Van Der Lei, B. (2018). Thread-lift sutures: still in the lift? A systematic review of the literature. Plastic and Reconstructive Surgery, 141(3), 341-347.

Hammoudeh, Z. S., \& Stevens, W. G. (2019). Nonsurgical Adjuncts Following Facelift to Achieve Optimal Aesthetic Outcomes:" Icing on the Cake". Clinics in Plastic Surgery, 46(4), 613-623.

Jang, H. J., Lee, W. S., Hwang, K., Park, J. H., \& Kim, D. J. (2005). Effect of cog threads under rat skin. Dermatologic Surgery, 31(12), 1639. 1644.

Kang, S. H., Moon, S. H., Rho, B. I., Youn, S. J., \& Kim, H. S. (2019). Wedge-shaped polydioxanone threads in a folded configuration ("Solid fillers"): A treatment option for deep static wrinkles on the upper face. Journal of Cosmetic Dermatology, 18(1), 65-70.

Lycka, B., Bazan, C., Poletti, E., \& Treen, B. (2004). The emerging technique of the antiptosis subdermal suspension thread. Dermatologic Surgery, 30(1), 41-44.

Matarasso, A. (2013). Introduction to the barbed sutures supplement: the expanding applications of barbed sutures. Aesthetic Surgery Journal, 33(Suppl), 7S-11S.

Mulholland, R. S. (2014). Nonexcisional, minimally invasive rejuvenation of the neck. Clinics in Plastic Surgery, 41(1), 11-31.

O'Connell, J. B. (2015). Rhytidectomy utilizing bidirectional self-retaining sutures: the bidirectional lift and the extended bidirectional lift. Aesthetic surgery journal, 35(6), 633-643.

Obagi, S., \& Willis, C. (2018). Autologous fat augmentation of the face. Atlas of the Oral and Maxillofacial Surgery Clinics of North America, 26(1), 41-50.
Philipp-Dormston, W. G., Schuster, B., \& Podda, M. (2020). Perceived naturalness of facial expression after hyaluronic acid filler injection in nasolabial folds and lower face. Journal of Cosmetic Dermatology, 19(7), 1600-1606.

Rachel, J. D., Lack, E. B., \& Larson, B. (2010). Incidence of complications and early recurrence in 29 patients after facial rejuvenation with barbed suture lifting. Dermatologic Surgery, 36(3), 348-354.

Raspaldo, H., Baspeyras, M., Bellity, P., Dallara, J. M., Gassia, V., Niforos, F. R., ... \& Consensus Group. (2011). Upper-and mid-face anti-aging treatment and prevention using onabotulinumtoxin A: the 2010 multidisciplinary French consensus-part 1. Journal of Cosmetic Dermatology, 10(1), 36-50.

Rezaee Khiabanloo, S., Jebreili, R., Aalipour, E., Eftekhari, H., Saljoughi, N., \& Shahidi, A. (2019). Innovative techniques for thread lifting of face and neck. Journal of Cosmetic Dermatology, 18(6), 1846-1855.

Rodriquez-Bruno, K., \& Papel, I. D. (2011). Rhytidectomy: principles and practice emphasizing safety. Facial Plastic Surgery, 27(01), 98-111.

Rohrich, R. J., Rios, J. L., Smith, P. D., \& Gutowski, K. A. (2006). Neck rejuvenation revisited. Plastic and Reconstructive Surgery, 118(5), 1251-1263.

Sarigul Guduk, S., \& Karaca, N. (2018). Safety and complications of absorbable threads made of poly-L-lactic acid and poly lactide/glycolide: experience with 148 consecutive patients. Journal of Cosmetic Dermatology, 17(6), 1189-1193.

Savoia, A., Accardo, C., Vannini, F., Di Pasquale, B., \& Baldi, A. (2014). Outcomes in thread lift for facial rejuvenation: a study performed with happy lift ${ }^{\mathrm{TM}}$ revitalizing. Dermatology and Therapy, 4(1), 103114.

Scharffetter-Kochanek, K., Brenneisen, P., Wenk, J., Herrmann, G., Ma, W., Kuhr, L., ... \& Wlaschek, M. (2000). Photoaging of the skin from phenotype to mechanisms. Experimental Gerontology, 35(3), $307-$ 316.

Silva-Siwady, J. G., Díaz-Garza, C., \& Ocampo-Candiani, J. (2005). A case of Aptos thread migration and partial expulsion. Dermatologic Surgery, 31(3), 356-358.

Smith, R. M., \& Papel, I. D. (2018). Difficult necks and unresolved problems in neck rejuvenation. Clinics in Plastic Surgery, 45(4), 611622.

Suh, D. H., Jang, H. W., Lee, S. J., Lee, W. S., \& Ryu, H. J. (2015). Outcomes of polydioxanone knotless thread lifting for facial rejuvenation. Dermatologic Surgery, 41(6), 720-725.

Sulamanidze, M. A., Fournier, P. F., Paikidze, T. G., \& Sulamanidze, G. M. (2002). Removal of facial soft tissue ptosis with special threads. Dermatologic Surgery, 28(5), 367-371.

Tan, M., \& Kontis, T. C. (2015). Midface volumization with injectable fillers. Facial Plastic Surgery Clinics, 23(2), 233-242.

Uitto, J., Matsuoka, L. Y., \& Kornberg, R. L. (1986). Elastic fibers in cutaneous elastoses. In: Rudolph, R. (eds) Problems in Aesthetic Surgery: Biological Causes and Clinical Solutions (pp. 307-338). St Louis.

Cite as: Celik, N. (2020). The new era in office-based facial rejuvenation: Promising technology of silicone threads. Front Life Sci RT, 2(1), 30-34. 